\title{
Liver Resection for Hepatocellular Carcinoma and the Barcelona Clinic Liver Cancer Criteria: Is It Time to Push the Limits?
}

\author{
Leon Naar, MD, and Ioannis Hatzaras, MD, MPH, PhD, FACS \\ Fourth Department of Surgery, Attikon University Hospital, National and Kapodistrian University of Athens, Athens, \\ Greece
}

When the Barcelona Clinic Liver Cancer (BCLC) criteria and guidelines were implemented (1999), our knowledge of the natural history of hepatocellular carcinoma (HCC) was limited. ${ }^{1}$ Particularly, little was known regarding patient and tumor characteristics that could affect prognosis, and even less knowledge was available to guide a surgeon's selection of patients to resect. At that time, available oncologic research evidence regarding $\mathrm{HCC}$ survival was coming either from analyses of patients who were not treated, for a variety of reasons, or from retrospective studies including large numbers of patients who predominantly had advanced-stage disease. ${ }^{1}$

The BCLC criteria were not based on a prognostic model derived from the statistical analysis of a specific HCC cohort, but instead were author-derived and based on studies of patients with similar baseline and tumor characteristics. Therefore, the authors of the BCLC criteria took a position of safety and proposed surgical resection only for early HCC (stages 0 and A). Transarterial chemoembolization (TACE) or chemotherapy was recommended to treat patients with intermediate to advanced HCC (stages B and $\mathrm{C}$ ), and palliative/symptomatic-only supportive treatment was used for patients with end-stage disease (stage D) ${ }^{1,2}$ In 2006, the same group performed a prospective validation of the BCLC criteria, proving their predictive power. ${ }^{3}$ Although the BCLC criteria were developed initially for cirrhotic HCC patients, providers have adopted

(C) Society of Surgical Oncology 2020

First Received: 25 March 2020;

Published Online: 15 April 2020

I. Hatzaras, MD, MPH, PhD, FACS

e-mail: info@hatzaras.gr them to stratify non-cirrhotic HCC patients. Several studies since the original report have consistently shown that the BCLC criteria offer the best survival prediction model. ${ }^{3-5}$

In the 2 decades since inception of the BCLC criteria, surgical techniques for liver resection have evolved considerably, resulting in significantly decreased operative mortality and postoperative morbidity and mortality rates. ${ }^{6-8}$ The authors of a study published in 1995 that investigated liver resection for HCC noted $32 \%, 4.5 \%$, and $6 \%$ morbidity, operative, and in-hospital mortality rates, respectively. ${ }^{6}$ In 2020 , several large-volume hepatobiliary centers exist, staffed with highly experienced surgeons. The current morbidity and mortality rates after liver resection are closer to $15 \%$ and $1 \%$, respectively. ${ }^{7,8}$ The increased safety of liver resection has resulted in an ongoing interest to expand surgical management for BCLC $\mathrm{B}$ and $\mathrm{C}$ patients. Despite the wide adoption of the BCLC criteria by the surgical community, recent studies have shown improved perioperative outcomes and long-term survival for sporadic BCLC B and C patients managed surgically. ${ }^{9-12}$ However, available evidence on recurrence patterns and survival of patients undergoing resection beyond the BCLC guidelines is lacking.

In a landmark study by Tsilimigras et al. ${ }^{13}$ published in the Annals this month, the authors assessed one of the largest cohorts of HCC patients who underwent resection using an international, multi-institutional database. They provided a contemporary analysis of HCC patients within and beyond the BCLC system who underwent operative management. The multi-institutional nature of the current study is particularly important for the generalizability of their results. Briefly, the study included 756 patients undergoing hepatectomy for HCC, $20 \%$ of whom were beyond the BCLC criteria. One-third of the patients had a major hepatectomy, and one-fourth of all the patients had a 
minimally invasive hepatectomy. Interestingly, the serious morbidity (Clavien-Dindo classification $\geq 3$ ) and mortality in the two groups were very low, and statistically insignificant in comparison. After a median 3-year followup period, the recurrence rates were naturally found to be higher for the patients who had surgery beyond the BCLC criteria $(59.7 \%$ vs $45.5 \%$; $p=0.002)$. However, the incidence of true local margin recurrence was low $(5.5 \%)$ and statistically not significant in either group. In fact, the majority of patients had intrahepatic recurrence (within BCLC $74.3 \%$ vs. beyond BCLC $70.8 \% ; p=0.80$ ). Notably, the highest incidence of recurrence was within the first year $(21.3 \%$ within BCLC criteria and $38.3 \%$ beyond BCLC criteria), which highlights the importance of robust surveillance of patients undergoing resection for HCC, especially during the first 24 months after resection. ${ }^{14}$ Prognostication with regression analysis for BCLC 0 and A patients identified a-fetoprotein (AFP) level higher than $400 \mathrm{ng} / \mathrm{mL}$, poor differentiation, liver capsule involvement, and R1 resection as independent predictors of postoperative recurrence. In contrast, only an AFP level higher than $400 \mathrm{ng} / \mathrm{mL}$ and $\mathrm{R} 1$ resection were associated with a higher risk of recurrence in BCLC B/C patients undergoing surgery.

The higher rate of recurrence among the patients undergoing resection beyond the BCLC criteria in the Tsilimigras et al. ${ }^{13}$ study is obvious and expected, raising questions about offering hepatectomy to BCLC B and C patients. However, based on the results of their study, it is important to underscore that the majority of the recurrences were de novo intrahepatic lesions and not systemic disease. This pattern of recurrence, however, offers therapeutic opportunities for additional liver-directed interventions, including re-do hepatectomy, ablative techniques, transarterial embolization (TAE), and/or TACE. The lower overall survival (OS) rates for BCLC B and C patients managed with liver resection in the Tsilimigras et al. ${ }^{13}$ study are, from an oncologic standpoint, not surprising. However, they should be considered in a different perspective and compared instead with the OS of BCLC B and $\mathrm{C}$ patients who are not managed with a hepatectomy but instead undergo ablation, TACE, or sorafenib treatment per the BCLC recommendations. With this in mind, a $50 \%$ 5-year OS for BCLC B and C patients who undergo resection is more than acceptable considering that OS of BCLC B is 16 months for untreated patients and 40 months for well-selected patients who undergo TACE. ${ }^{15}$ Moreover, for patients with BCLC C, the median survival time ranges between 6 and 12 months in the literature. ${ }^{15-18}$

In summary, the BCLC criteria were described and validated 20 years ago. In the interim, hepatobiliary surgery has evolved significantly. The main treatment method for HCC, liver resection, currently is much safer and more available for patients. Currently, surgeons more commonly offer a minimally invasive option to their patients, expediting their recovery. As such, the BCLC system should be revised to reflect modern liver surgery safety standards, and BCLC stages B and C should not be considered as absolute contraindications to surgery. Patients who harbor a low volume of disease with a low AFP should be considered for hepatectomy. The axiom "good patient selection leads to good results" is as relevant as ever.

\section{FUNDING None}

DISCLOSURE There are no conflicts of interest.

\section{REFERENCES}

1. Llovet JM, Bru C, Bruix J. Prognosis of hepatocellular carcinoma: the BCLC staging classification. Semin Liver Dis. 1999;19:329-38.

2. Befeler AS, Di Bisceglie AM. Hepatocellular carcinoma: diagnosis and treatment. Gastroenterology. 2002;122:1609-19.

3. Cillo U, Vitale A, Grigoletto F et al. Prospective validation of the Barcelona Clinic Liver Cancer staging system. J Hepatol. 2006;44:723-31.

4. Marrero JA, Fontana RJ, Barrat A et al. Prognosis of hepatocellular carcinoma: comparison of 7 staging systems in an American cohort. Hepatology. 2005;41:707-16.

5. Cillo U, Bassanello M, Vitale A et al. The critical issue of hepatocellular carcinoma prognostic classification: which is the best tool available? J Hepatol. 2004;40:124-31.

6. Lai EC, Fan ST, Lo CM et al. Hepatic resection for hepatocellular carcinoma: an audit of 343 patients. Ann Surg. 1995;221:291-8.

7. Guro H, Cho JY, Han HS et al. Current status of laparoscopic liver resection for hepatocellular carcinoma. Clin Mol Hepatol. 2016;22:212-8.

8. Yamazaki S, Takayama T. Management strategies to minimize mortality in liver resection for hepatocellular carcinoma. Jpn J Clin Oncol. 2017;47:899-908.

9. Wada H, Eguchi H, Noda $\mathrm{T}$ et al. Selection criteria for hepatic resection in intermediate-stage (BCLC stage B) multiple hepatocellular carcinoma. Surgery. 2016;160:1227-35.

10. Bhandare MS, Patkar S, Shetty N et al. Liver resection for HCC outside the BCLC criteria. Langenbecks Arch Surg. 2018;403:37-44.

11. Moris D, Felekouras E. Ignore reality but not the consequences of its ignorance: broaden guidelines in surgery of hepatocellular carcinoma. Hepatology. 2017;65:1772-3.

12. Guo H, Wu T, Lu Q et al. Surgical resection improves long-term survival of patients with hepatocellular carcinoma across different Barcelona Clinic Liver Cancer stages. Cancer Manag Res. 2018;10:361-9.

13. Tsilimigras D, Bagante F, Moris D et al. Recurrence patterns and outcomes after resection of hepatocellular carcinoma within and beyond the Barcelona Clinic Liver Cancer Criteria. Ann Surg Oncol. 2020; Epub ahead of print.

14. Hatzaras I, Bischof DA, Fahy B et al. Treatment options and surveillance strategies after therapy for hepatocellular carcinoma. Ann Surg Oncol. 2014;21:758-66.

15. European Association for the Study of the Liver. European association for the study of the, EASL clinical practice 
guidelines: management of hepatocellular carcinoma. $J$ Hepatol. 2018;69:182-236.

16. Pawlik TM, Poon RT, Abdalla EK et al. Critical appraisal of the clinical and pathologic predictors of survival after resection of large hepatocellular carcinoma. Arch Surg. 2005;140:450-7.

17. $\mathrm{Ng} \mathrm{KK}$, Vauthey JN, Pawlik TM et al. Is hepatic resection for large or multinodular hepatocellular carcinoma justified? Results from a multi-institutional database. Ann Surg Oncol. 2005;12:364-73.
18. Pandey D, Lee KH, Wai CT, Wagholikar G, Tan KC. Long-term outcome and prognostic factors for large hepatocellular carcinoma (10 cm or more) after surgical resection. Ann Surg Oncol. $2007 ; 14: 2817-23$.

Publisher's Note Springer Nature remains neutral with regard to jurisdictional claims in published maps and institutional affiliations. 